\title{
Expression of aromatase and estrogen receptor alpha in chondrosarcoma, but no beneficial effect of inhibiting estrogen signaling both in vitro and in vivo
}

Danielle Meijer ${ }^{1}$, Hans Gelderblom², Marcel Karperien ${ }^{3}$, Anne-Marie Cleton-Jansen ${ }^{1}$, Pancras CW Hogendoorn ${ }^{1}$ and Judith VMG Bovée ${ }^{1 *}$

\begin{abstract}
Background: Chondrosarcomas are malignant cartilage-forming tumors which are highly resistant to conventional chemotherapy and radiotherapy. Estrogen signaling is known to play an important role in proliferation and differentiation of chondrocytes and in growth plate regulation at puberty. Our experiments focus on unraveling the role of estrogen signaling in the regulation of neoplastic cartilage growth and on interference with estrogen signaling in chondrosarcomas in vitro and in vivo.

Methods: We investigated the protein expression of estrogen receptor alpha (ESR1), androgen receptor (AR), and aromatase in tumor specimens of various chondrosarcoma subtypes, and (primary) chondrosarcoma cultures. Doseresponse curves were generated of conventional central chondrosarcoma cell lines cultured in the presence of $17 \beta$-estradiol, dihydrotestosterone, 4-androstene-3,17 dione, 4-hydroxytamoxifen, fulvestrant and aromatase inhibitors. In a pilot series, the effect of anastrozole $(n=4)$ or exemestane $(n=2)$ treatment in 6 chondrosarcoma patients with progressive disease was explored.

Results: We showed protein expression of ESR1 and aromatase in a large majority of all subtypes. Only a minority of the tumors showed few AR positive cells. The dose-response assays showed no effect of any of the compounds on proliferation of conventional chondrosarcoma in vitro. The median progression-free survival of the patients treated with aromatase inhibitors did not significantly deviate from untreated patients.

Conclusions: The presence of ESR1 and aromatase in chondrosarcoma tumors and primary cultures supports a possible role of estrogen signaling in chondrosarcoma proliferation. However, our in vitro and pilot in vivo studies have shown no effect of estrogen-signaling inhibition on tumor growth.
\end{abstract}

\section{Background}

Chondrosarcomas of bone are malignant cartilage-forming tumors which are highly resistant to conventional chemotherapy and radiotherapy $[1,2]$. However, recently various promising targets were discovered and the exploration of suitable therapies continues $[3,4]$. Conventional chondrosarcomas represent about $90 \%$ of all chondrosarcomas. Most conventional chondrosarcomas

\footnotetext{
* Correspondence: J.V.M.G.Bovee@lumc.nl

'Department of Pathology, Leiden University Medical Center, Leiden, The Netherlands

Full list of author information is available at the end of the article
}

are located in the medullar cavity of the bone and are called central chondrosarcoma. About $15 \%$ of conventional chondrosarcomas arise from the surface of bone and are designated as peripheral chondrosarcomas. Conventional chondrosarcomas often show local destructive growth and the high-grade tumors commonly metastasize [5].

Besides conventional chondrosarcoma, several rare chondrosarcoma subtypes are defined, together constituting $10-15 \%$ of all chondrosarcomas. Dedifferentiated chondrosarcoma (10\%) is a tumor containing a highgrade dedifferentiated non-cartilaginous sarcoma next to

\section{Biomed Central}


a usually low-grade malignant well-differentiated cartilage-forming tumor, with a sharply defined junction between the two components. It bears a poor prognosis and no targets for therapy have been reported so far [6]. Mesenchymal chondrosarcoma (2\%) is a highly malignant lesion occurring in the bone and soft tissue of relatively young patients. The tumor consists of differentiated cartilage mixed with undifferentiated small round cells and usually follows an aggressive course with a high rate of distant metastases, and a 5-year overall survival of 55\% [7]. Clear cell chondrosarcoma (2\%) is a low-grade malignant tumor, which rarely metastasizes, but commonly recurs after curettage. About 15\% of the patients die as a result of the disease [8]. The lack of efficacious treatment for all different subtypes of chondrosarcomas emphasizes the need to identify new treatment strategies.

One of the potential targets for therapy is the estrogen-signaling pathway. Mutations in ESR1 and CYP19A1, the gene for aromatase, demonstrated an important role for estrogen in the proliferation and differentiation of chondrocytes in the epiphyseal growth plate [9]. Estrogen induces the pubertal growth spurt, and at the end of puberty growth plate fusion [10]. Furthermore, osteochondromas, the benign precursors of peripheral chondrosarcomas, stop growing at the end of puberty, suggesting an inhibitory effect of estrogens on these tumors. In addition, ESR1 and ESR2 expression has been shown to be a common phenomenon in chondrosarcomas [11,12]. In a previous study, our group also demonstrated functional activity of the estrogen-producing enzyme aromatase in chondrosarcoma cells in vitro [11]. These results indicated that the ESR signaling pathway might be a potential target for endocrine treatment of metastatic or irresectable chondrosarcoma.

For already three decades endocrine therapy plays a crucial role in the treatment of women with hormoneresponsive breast cancer. Breast cancer and chondrosarcomas were found to occur relatively frequently in the same patient. A population-based study by Odink et al. implicated a 7.62 times increased risk for the same female patient to have both breast cancer and a cartilaginous tumor [13]. The mean age of onset in patients with breast cancer as the first tumor and chondrosarcoma as a second tumor is nearly 10 years earlier than breast cancer in general [13]. These observations may suggest a genetic trait. Remarkably, the expression of ESR1 was significantly higher in breast cancer associated with chondrosarcoma [14].

The two strategies used for endocrine treatment are blockade of ESR1 using selective estrogen receptor modulators/downregulators like tamoxifen and fulvestrant, and deprivation of estrogen production by inhibiting aromatase with anastrozole, letrozole, and exemestane.
In our above-mentioned study, we showed that the aromatase activity and proliferation of chondrosarcoma cells slightly decreased after addition of the aromatase inhibitor exemestane [11]. In our present study, we focused on further unraveling the role of estrogen in the regulation of neoplastic cartilage growth in a larger cohort of various chondrosarcoma subtypes, including conventional central and peripheral chondrosarcoma as well as dedifferentiated, mesenchymal, and clear cell chondrosarcoma. Moreover, using a larger set of drugs targeting the estrogen-signaling pathway we investigated whether interference with estrogen signaling could inhibit chondrosarcoma growth. We aimed to validate and expand our previous in vitro data by measuring the effects of estrogens, androgens, tamoxifen, fulvestrant, and aromatase inhibitors on the proliferation of various chondrosarcoma cell cultures. Furthermore, we explored the efficacy of aromatase inhibitors in a set of patients with metastatic or locally advanced chondrosarcoma.

\section{Methods}

\section{Tumor tissue}

All specimens in this study were handled according to the ethical guidelines described in "Code for Proper Secondary Use of Human Tissue in The Netherlands" of the Dutch Federation of Medical Scientific Societies. Conventional central and peripheral chondrosarcoma, and the rare subtypes dedifferentiated, mesenchymal, and clear cell chondrosarcoma were selected based on accepted clinicopathological and radiological criteria [15]. In total, formalin-fixed paraffin-embedded (FFPE) specimens from 175 patients, including the 6 patients in our pilot study, were collected from the archives of the Department of Pathology, LUMC, The Netherlands $(n=100)$, Nuffield Department of Orthopaedic Surgery, University of Oxford, UK $(n=7)$, Institute of Orthopaedics and Musculoskeletal Science, UCL, UK $(\mathrm{n}=22)$, Laboratory of Oncologic Research, ROI, Italy $(\mathrm{n}=30)$, Department of Pathology, RH, Denmark ( $\mathrm{n}=$ 9), Department of Pathology, Medizinische Universität Graz, Austria $(n=7)$. All were primary tumors except for three clear cell chondrosarcomas and six mesenchymal chondrosarcomas, from which only recurrences were available. Clinicopathological data are shown in table 1. Histological grading was performed according to Evans [5].

\section{Tissue microarray (TMA) construction}

Of the rare chondrosarcoma subtypes we constructed TMAs using a TMA Master (3DHISTECH Ltd, Budapest, Hungary). TMAs contained $2 \mathrm{~mm}$ cores of each sample, in triplicate. From the dedifferentiated chondrosarcomas we included both the well differentiated and the dedifferentiated components. The clinical details are 
Table 1 Clinicopathological data of the 175 formalin-fixed paraffin-embedded cartilaginous tumors

\begin{tabular}{llllllll}
\hline & EC & CS & OC & PCS & DDCS & CCS & MCS \\
\hline Total number of tumors & $\mathbf{3}$ & $\mathbf{4 6}$ & $\mathbf{1 0}$ & $\mathbf{2 8}$ & $\mathbf{4 2}$ & $\mathbf{2 3}$ & $\mathbf{2 3}$ \\
Grade I & - & 16 & - & 12 & - & - & - \\
Grade II & - & 18 & - & 13 & - & - & - \\
Grade III & - & 12 & - & 3 & - & - & 8 \\
Male & 1 & 22 & 6 & 17 & 21 & 17 & 15 \\
Female & 2 & 23 & 4 & 10 & 21 & - \\
Enchondromatosis/MO & 0 & 1 & 5 & 10 & - & - & - \\
Median age yrs (range) & $38(37-50)$ & $51(20-79)$ & $14.5(6-24)$ & $38(16-61)$ & $66(26-85)$ & $43(20-79)$ & $29.5(15-70)$ \\
\hline
\end{tabular}

Abbreviations: enchondroma (EC), conventional central and peripheral chondrosarcoma (CS and PCS), osteochondroma (OC), dedifferentiated chondrosarcoma (DDCS), clear cell chondrosarcoma (CCS), and mesenchymal chondrosarcoma (MCS).

outlined in Table 1. Normal non-decalcified liver, kidney, and tonsil samples were included on the TMAs for orientation purposes and as internal positive controls.

\section{Immunohistochemistry (IHC)}

Details of the primary antibodies used for immunohistochemistry are described in Table 2. As negative controls, slides were incubated in PBS/BSA $1 \%$ without primary specific antibodies. AR and aromatase immunohistochemical stainings of the patient material were semiquantitatively scored for nuclear and cytoplasmic staining respectively. Scores were given for intensity $(0=$ absent, 1 = weak, $2=$ moderate, $3=$ strong) and for the percentage of positive cells $(0=0 \%, 1=1-24 \%, 2=25-49 \%, 3=50$ $74 \%$, and $4=75 \%-100 \%$ ). To avoid tumors with single positive cells being regarded as positive, a cut-off level of a total sum $\geq 4$ was applied. ESR1 was scored for nuclear staining, with positivity defined as $\geq 10 \%$ (weakly) positive cells, according to standard clinical procedures for scoring ESR1-positive breast cancer [16,17]. Scoring was performed by two independent observers without knowledge of the clinicopathological data. For dedifferentiated chondrosarcoma, the well differentiated and the dedifferentiated component were scored separately. Likewise, for mesenchymal chondrosarcoma both the cartilaginous areas and the small cell component were evaluated.

\section{Cell cultures and conditions}

Chondrosarcoma cell lines SW1353 (ATCC, Manassas, VA), OUMS27 [18], CH2879 [19], and JJ012 [20], and breast cancer cell line ZR-75-1 [21] were cultured in RPMI 1640 supplemented with 10\% heat-inactivated fetal bovine serum (FBS) (Gibco, Invitrogen Life-Technologies,
Scotland, UK) (Table 3). ZR-75-1 cultures were additionally supplemented with $1 \mathrm{nmol} / \mathrm{L} 17 \beta$-estradiol $\left(\mathrm{E}_{2}\right)$ (Sigma). Two chondrosarcoma primary cultures, L835 and L869, were generated as described previously [11] and were cultured in collagen I-coated culture flasks in RPMI 1640 supplemented with $20 \%$ heat-inactivated fetal calf serum (Invitrogen), 2\% penicillin/streptomycin (MP Biomedicals), and 1\% glutamax (Invitrogen) (Table 3). Cells were grown at $37^{\circ} \mathrm{C}$ in a humidified incubator with $95 \%$ air and $5 \% \mathrm{CO}_{2}$. The primary chondrosarcoma cultures expressed mRNA of at least two of the cartilaginous markers collagen 2, collagen 10, aggecan or SOX9 [11]. In addition, karyotyping of L835 and L869 by COBRA-FISH showed an aberrant number of chromosomes, thereby confirming their tumorigenic origin.

\section{Protein detection in chondrosarcoma cell cultures}

Four T75 culture flasks of 4 chondrosarcoma cell lines (SW1353, CH2879, OUMS27, JJ012) and 2 primary chondrosarcoma cell cultures (L869 and L835), and a positive control (ZR-75-1) were trypsinized and washed twice with cold PBS. Cells were formalin-fixed over night and subsequently embedded in paraffin. Using IHC, we determined ESR1 protein expression, as described above.

\section{Proliferation assays}

To monitor the effects of the estrogen signaling pathway on chondrosarcoma cell proliferation we performed various experiments. An overview of all different conditions tested is given in Table 4. For the WST-1 assays with steroids and inhibitors, SW1353, CH2879, OUMS27, JJ012, L869, and L835 cells were seeded into collagen I-

Table 2 Procedures and details of the primary antibodies used for immunohistochemistry

\begin{tabular}{|c|c|c|c|c|c|c|c|}
\hline Protein & Origin & Number & Dilution & Species & Antigen retrieval & Blocking & Positive control \\
\hline$\overline{\text { ESR1 }}$ & Invitrogen/zymed & $18-0174 Z$ & $1: 200$ & Rabbit & Tris-EDTA & $30^{\prime}$ 5\% ELK milk & breast cancer \\
\hline aromatase & Abcam & Ab18995 & $1: 300$ & Rabbit & Citrate & 30' 5\% ELK milk & placenta \\
\hline$A R$ & Dako & AR441 & $1: 200$ & Mouse & Tris-EDTA & - & cervix stroma \\
\hline
\end{tabular}


Table 3 Chondrosarcoma cultures

\begin{tabular}{lllllll}
\hline & Sample & Type & Grade & Gender & Age & Passage \\
\hline 1 & SW1353 & Cell line & II & F & 72 & 18 \\
2 & CH2879 & Cell line & III & F & 35 & 31 \\
3 & OUMS27 & Cell line & III & M & Na & 22 \\
4 & Jj012 & Cell line & II & M & 39 & 10 \\
5 & L835 & Primary culture & III & M & 55 & 15 \\
6 & L869 & Primary culture & ॥ & M & 52 & 18 \\
\hline
\end{tabular}

coated 96 wells plates (BD Biosciences) at a density of 1500 cells per well for the SW1353 and JJ012 cell lines and 5000 cells per well for the other cultures. The cells were plated in phenol red-free RPMI 1640 medium (Invitrogen) supplemented with 10\% heat-inactivated charcoal-stripped FBS (Invitrogen). After 24 hours, serial dilutions of the steroids $17 \beta$-estradiol (Sigma), 4-androstene-3,17-dione (Sigma) and dihydrotestosterone (Fluka Analytical) (100 pM-1 $\mu \mathrm{M})$, anti-steroids 4-hydroxytamoxifen (Sigma) and fulvestrant (Sigma) $(1 \mathrm{nM}-10 \mu \mathrm{M})$, aromatase inhibitors anastrozole, letrozole and exemestane $(1 \mathrm{nM}-10 \mu \mathrm{M})$ or combinations were added. The compounds were solved in ethanol. As vehicle control, ethanol was added with concentrations never exceeding $0.1 \%$. All concentrations were tested at least in quadruplicate in a total volume of $100 \mu \mathrm{l}$. After 3 days, $10 \mu \mathrm{l}$ proliferation reagent WST-1 (Roche Diagnostics) were added to each well, and the cells were returned to the incubator for three hours. Absorbance was measured at $450 \mathrm{~nm}$ with a Victor ${ }^{3}$ Multilabel Counter 1420-042 (Perkin Elmer, MA, USA). Values were corrected for background, averaged and normalized to the vehiclecontrol cultures. FBS dependence was tested likewise for SW1353, CH2879, and OUMS27. For cell counting experiments, 24000 cells were seeded in a 24 wells plate. The experimental set up was identical to the proliferation assays with a total volume of $1 \mathrm{ml}$. Cells were counted after 3 and 7 days of treatment according to experiments previously published by our group $[3,11]$.

\section{Patients}

Five patients with grade II or III conventional chondrosarcoma and one patient with dedifferentiated chondrosarcoma were treated with the aromatase inhibitors anastrozole $1 \mathrm{mg}$ once daily (4 patients, including the patient with dedifferentiated chondrosarcoma) and exemestane $25 \mathrm{mg}$ once daily ( 2 patients). Median age was 44 years (range 33-68); 3 patients had metastatic disease and 3 had locally advanced tumors. Tumor measurements and response evaluations were performed according to RECIST [22]. From the patients with conventional chondrosarcoma, FFPE tumor specimens were stained for ESR1 and aromatase protein.

\section{Results}

Expression of ESR1, aromatase, and AR in FFPE chondrosarcoma tumor specimens

Results of ESR1, aromatase, and AR immunohistochemical stainings on 175 FFPE tumor specimens are shown in Table 5. Expression for ESR1 and aromatase was detected in the majority of all subtypes (Table 5 and Figure 1). In conventional central and peripheral chondrosarcoma we observed immunoreactivity against ESR1 in $81 \%$ (34 out of 42 ) and $81 \%$ (21 out of 26 ) of the tumors, respectively. We observed ESR1 in $73 \%$ of the well differentiated and in $84 \%$ of the dedifferentiated component of dedifferentiated chondrosarcoma. Positive staining of the two components was strongly correlated. In mesenchymal chondrosarcomas, $67 \%$ of the small cell components were positive, versus $33 \%$ of the cartilaginous areas. Only a few strongly AR positive cells were detected in a minority of the chondrosarcomas of various subtypes. Aromatase protein, the enzyme responsible for the conversion of androstenedione and androgens to estrogens, was expressed in $86 \%$ (38 out of 44 ) and $93 \%$ (25 out of 27) of the central and peripheral chondrosarcomas respectively. Almost all well differentiated (97\%) and dedifferentiated (89\%) components of dedifferentiated chondrosarcoma were positive for aromatase. Of the cartilaginous area of mesenchymal chondrosarcoma $77 \%$ showed aromatase positivity versus $52 \%$ of the small cell component. In central chondrosarcoma no correlation with histological grade was observed with any of the proteins. In peripheral chondrosarcoma only $33 \%$ of the grade III tumors showed

Table 4 Experimental conditions tested

\begin{tabular}{|c|c|}
\hline Test & Experimental conditions \\
\hline Steroids & E2 (Fig 2A), ASD, DHT (100 pM-1 $\mu \mathrm{M})$ \\
\hline Inhibitors of estrogen signaling & OHT, Fulvestrant, Anastrozole, Letrozole, Exemestane (1 nM-10 $\mu \mathrm{M})$ \\
\hline Steroids combined with inhibitors & E2 (1 nM) with OHT or Fulvestrant (1 nM-10 M) (Fig. 2B) \\
\hline FBS & $1 \%, 5 \%, 10 \% \mathrm{FBS}$ alone and combined with E2 or ASD (100 pM-1 $\mu \mathrm{M})$ \\
\hline Timepoints of measurements & 3 days, 7 days \\
\hline Methods of measurement & WST1 viability assay, cell counting \\
\hline
\end{tabular}

Abbreviations: 17ß-estradiol (E2), 4-androstene-3,17-dione, (ASD), dihydrotestosteron (DHT), 4- hydroxytamoxifen (OHT), Fetal bovine serum (FBS). 
Table 5 Immunohistochemical staining of 175 FFPE samples of chondrosarcoma patients

\begin{tabular}{lllllll}
\hline & ESR1 & & aromatase & AR* \\
\hline Enchondroma & $2 / 2$ & $100 \%$ & $3 / 3$ & $100 \%$ & $0 / 2$ & $0 \%$ \\
& & & & & & \\
Central CS & $34 / 42$ & $81 \%$ & $38 / 44$ & $86 \%$ & $6 / 41$ & $15 \%$ \\
grade I & $11 / 15$ & $73 \%$ & $13 / 15$ & $87 \%$ & $1 / 13$ & $8 \%$ \\
grade I| & $14 / 16$ & $88 \%$ & $15 / 17$ & $88 \%$ & $3 / 16$ & $19 \%$ \\
grade II & $9 / 11$ & $82 \%$ & $10 / 12$ & $83 \%$ & $2 / 12$ & $17 \%$ \\
& & & & & & \\
Osteochondroma & $5 / 8$ & $63 \%$ & $6 / 8$ & $75 \%$ & $1 / 7$ & $14 \%$ \\
& & & & & & \\
Peripheral CS & $21 / 26$ & $81 \%$ & $25 / 27$ & $93 \%$ & $4 / 28$ & $14 \%$ \\
grade | & $9 / 11$ & $82 \%$ & $11 / 12$ & $92 \%$ & $2 / 12$ & $17 \%$ \\
grade I| & $12 / 13$ & $92 \%$ & $12 / 13$ & $92 \%$ & $1 / 13$ & $8 \%$ \\
grade I|| & $1 / 3$ & $33 \%$ & $3 / 3$ & $100 \%$ & $1 / 3$ & $33 \%$
\end{tabular}

Dedifferentiated CS

well differentiated component $\quad 18 / 25 \quad 72 \% \quad 31 / 32 \quad 97 \% \quad 1 / 27 \quad 4 \%$ dedifferentiated component $\quad 30 / 35 \quad 86 \% \quad 34 / 38 \quad 89 \% \quad 2 / 37 \quad 5 \%$

Clear cell CS $\begin{array}{lllllll}15 / 22 & 69 \% & 15 / 22 & 69 \% & 0 / 22 & 0 \%\end{array}$

Mesenchymal CS

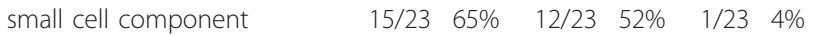

$\begin{array}{lllllll}\text { cartilaginous areas } & 5 / 15 & 33 \% & 10 / 13 & 77 \% & 0 / 12 & 0 \%\end{array}$

* In positive samples only few strongly positive cells were observed.

ESR1 expression. However, only three tumors were included in this group.

\section{Estrogen responsiveness of central chondrosarcoma in vitro}

SW1353, CH2879, OUMS27, JJ012, L869 and L835 were positive for ESR1 protein staining (Figure 1K, 1L and data not shown). Therefore, we investigated the effect of ESR-signaling modulation on the proliferation of chondrosarcoma cells in vitro by measuring the effect of steroids and clinical drugs inhibiting estrogen-signaling. We evaluated responsiveness of 4 central chondrosarcoma cell lines and 2 primary cultures to 3 different steroids (17 $\beta$-estradiol, the estrogen precursor androstenedione, and the non-aromatizable androgen dihydroxytestosterone). The proliferation of the cells was not significantly influenced by any of these factors in the chondrosarcoma cell lines and primary cultures, whereas a clear response was observed in the proliferation rate of the ZR-75-1 breast-cancer cell line, which was used as a positive control (Figure 2Aand data not shown). We also tested three aromatase inhibitors (anastrozole, letrozole, and exemestane), and two estrogen-receptor antagonists/downregulators (4-hydroxytamoxifen and fulvestrant) and again no effect was shown in the chondrosarcoma cell lines and cultures under the different conditions described in Table 4 (Figure 2Band data not shown).

\section{Clinical results}

In a pilot series, 6 consecutive patients with locally advanced or metastatic grade II or III conventional or dedifferentiated chondrosarcoma, for whom no standard treatment was available, were treated with aromatase inhibitors after informed consent. All tumors were radiologically progressive in the 6 months before initiation of therapy. All five conventional chondrosarcomas expressed ESR1 and aromatase protein, supporting the rationale of the treatment. The median progression-free survival was 5 months (range 4-10 months) in the conventional chondrosarcoma patients and 2 months in the patient with a metastatic dedifferentiated chondrosarcoma, which did not significantly deviate from untreated patients.

\section{Discussion}

Chondrosarcoma of bone is a malignant cartilage-forming tumor of which distinct clinical and histological subtypes are recognized. So far, for locally advanced and metastatic chondrosarcoma no treatment options are available. Previous studies have demonstrated the presence of the ESR1 and activity of aromatase in conventional chondrosarcoma $[11,12]$. Furthermore, in 2005, our group showed an effect of estrogens and the aromatase inhibitor exemestane on the proliferation of chondrosarcoma cells in vitro, indicating that chondrosarcomas might be susceptible to hormonal therapy. In that study, ESR 1 and CYP19A1 mRNA expression were demonstrated in a set of 23 conventional chondrosarcomas and 7 (primary) chondrosarcoma cultures. ESR1 protein expression was demonstrated in all 23 tumors tested. Addition of $17 \beta$ estradiol, 4-androstene-3,17-dione, and exemestane showed subtle effects on the proliferation of 2 cell cultures containing ESR1 and aromatase. After addition of 4-androstene-3,17-dione, an increase in proliferation was demonstrated. Proliferation was $131 \%$ of normal proliferation which decreased to $105 \%$ after inhibition with exemestane. A cell line lacking ESR1 and CYP19A1 did not show any response.

In the current study, we aimed to gain more insight into the possibility of treating chondrosarcoma patients with hormonal therapy by further investigating the expression of the hormone receptors ESR1 and AR, and of aromatase, the enzyme that mediates the last step in the biochemical formation of estrogen, in a larger set of conventional chondrosarcomas as well as three rare chondrosarcoma subtypes. In conventional chondrosarcoma, we furthermore monitored the effect of estrogen, 


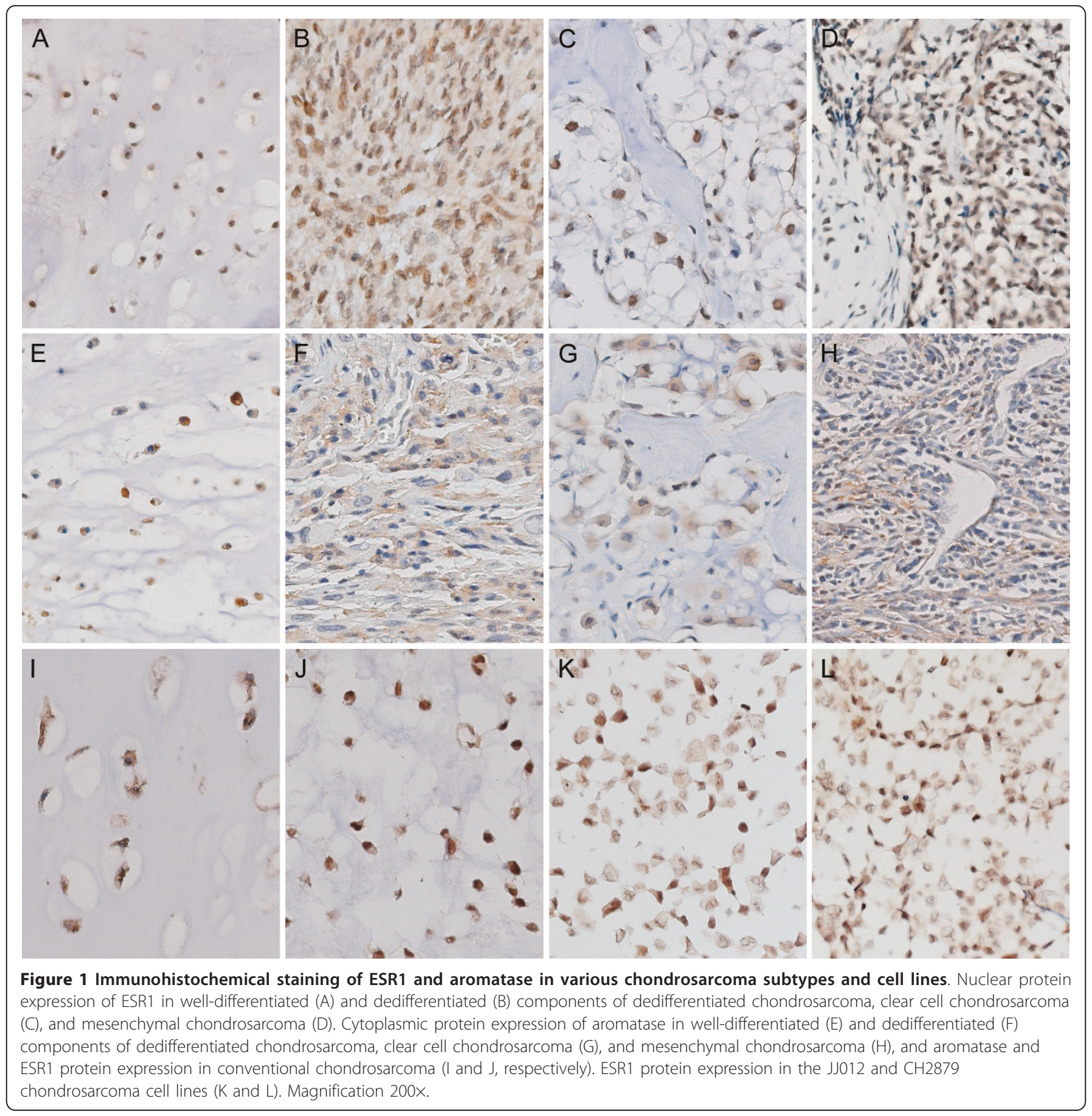

the estrogen precursor androstenedione and the nonaromatizable androgen dihydrotestosteron, and various known estrogen signaling-inhibiting drugs on the progression of chondrosarcoma cells in vitro.

We demonstrated expression of ESR1 in a large proportion of various types of cartilaginous tumors. In conventional central and peripheral chondrosarcoma we observed immunoreactivity against ESR1 in $81 \%$ (34 out of 42 ) and $81 \%$ (21 out of 26 ) of the tumors, respectively. These results confirm and extend 2 previous studies in which the authors demonstrated nuclear expression of ESR1 in subsets of 23 [11] and 31 [12] conventional chondrosarcomas. Grifone et al. [12] suggested a decrease or loss in ESR1 expression in the higher grade or dedifferentiated chondrosarcomas. We observed such a trend in the peripheral chondrosarcomas, where only $33 \%$ of the high grade tumors show positive staining for ESR1. However, this group included only three tumor specimens. In central chondrosarcoma no correlation with grade was observed. Aromatase protein, the enzyme responsible for the conversion of androstenedione and androgens to estrogens, was 


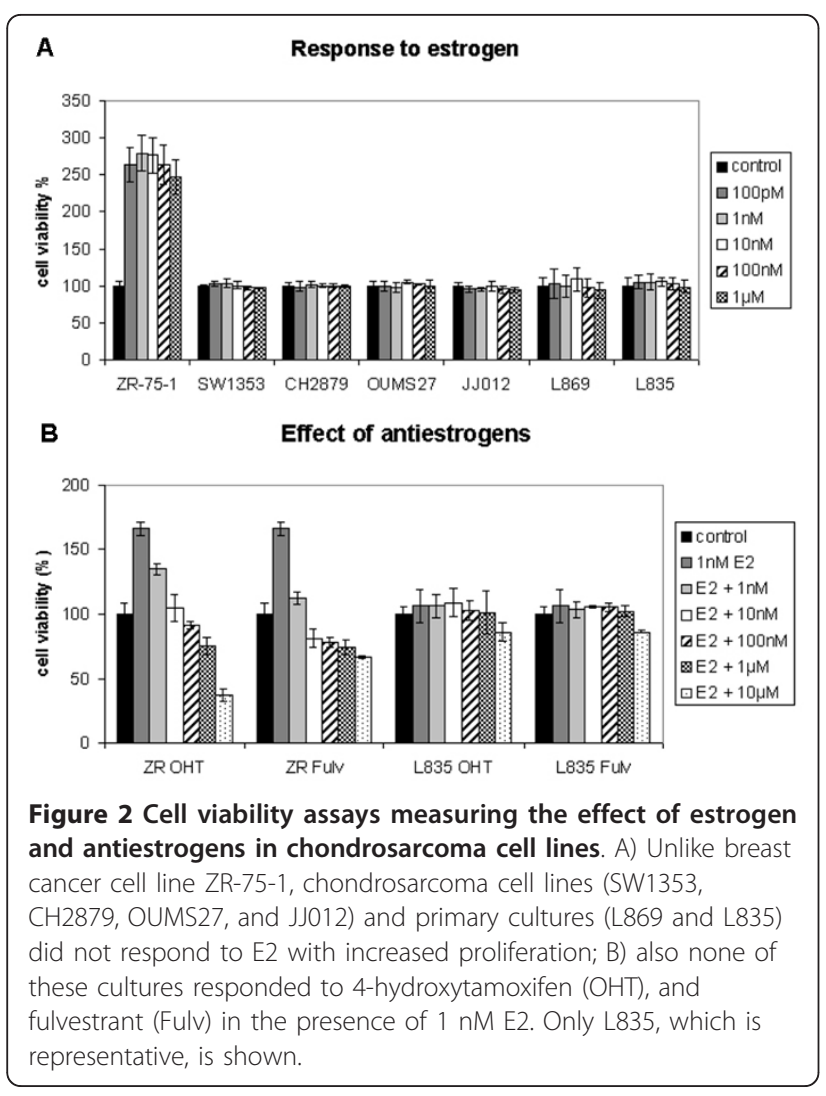

expressed in $86 \%$ and $93 \%$ of the central and peripheral chondrosarcomas respectively, suggesting that tumors are capable of metabolizing estrogens from precursors. AR is another important target for hormonal therapy in for example prostate cancer. As androstenedione is a steroid precursor for estrogens as well as androgens we also investigated the possibility of AR involvement in chondrosarcoma proliferation. However, AR nuclear protein expression was observed only in a small number of cases with very few positive cells.

Besides conventional chondrosarcoma, several rare chondrosarcoma subtypes are defined. Despite aggressive therapy, approximately $90 \%$ of the patients with dedifferentiated chondrosarcoma die with distant metastasis, within 2 years after diagnosis of the disease $[6,23]$. The low-grade component and the highly malignant component display ESR1 protein expression in $72 \%$ and $86 \%$ of the samples respectively. Aromatase was observed in 97\% and $89 \%$, suggesting the presence of estrogens.

Mesenchymal chondrosarcomas are usually very aggressive with a strong tendency of local recurrence and distant metastases. Patients have a 5-year overall survival of 55\% [7]. Although mesenchymal chondrosarcoma of bone is generally considered to lack sex predilection [24], Fanburg-Smith et al. [25] suggested a female predominance and raised the possibility of hormonal influence in the pathogenesis of this tumor. However, all their mesenchymal chondrosarcoma cases were ESR1 negative. In our study, in 65\% (15 out of 23) of the mesenchymal chondrosarcomas the small cell component was positive for ESR1, while in 33\% (5 out of 15) of the tumors also the cartilaginous areas were positive. Moreover, aromatase expression was observed in the small cells of $52 \%$ (12 out of 23 ) of the tumors, whereas the cartilage component demonstrated aromatase expression in $77 \%$ (10 out of 13). This might indicate that these tumors do have an active estrogen signaling pathway, which might be targetable by antiestrogens or aromatase inhibitors. Discrepant results may be explained by differences in ESR1 antibody and antigen retrieval protocols.

Clear cell chondrosarcoma is a low-grade variant of chondrosarcoma, which rarely metastasizes, but has a recurrence rate of $86 \%$ after curettage. About $15 \%$ of the patients die as a result of the disease [8]. We have observed ESR1 expression and aromatase expression each in $69 \%$ of the clear cell chondrosarcomas, suggesting that also these chondrosarcoma patients potentially might benefit from antiestrogen therapy and/or aromatase inhibition.

In vitro cell models to further study the effect of estrogen signaling on chondrosarcoma are available for conventional central chondrosarcoma only. No stimulation of proliferation of central chondrosarcoma cells was observed after addition of the non-aromatizable androgen dihydrotestosterone. This suggests no significant role for AR signaling in chondrosarcoma proliferation, which is consistent with the fact that very few tumors express AR.

In addition, in spite of positive immunohistochemical staining for ESR1 protein in all in vitro cell cultures, addition of $17 \beta$-estradiol, 4-androstene-3,17-dione or drugs targeting the estrogen-signaling pathway did not have a significant effect on the proliferation of the conventional central chondrosarcoma cell cultures. These results contradict our results published in 2005 , where proliferation was stimulated by $17 \beta$-estradiol and 4 -androstene-3,17dione, and inhibited by exemestane [11]. Although we included an identical experimental set up, cell culture conditions are never 100\% identical. For example, each batch of FBS contains different amounts of growth factors and other components which might influence experimental outcome. Also cell characteristics might have changed over time, resulting in passages insensitive to (anti)estrogens and aromatase inhibitors, as has been described before for certain breast cancer cell lines [26-28].

Breast cancer cell line ZR-75-1 is known to be completely dependent on estrogens for its proliferation, and proliferation can be fully inhibited by abrogating the estrogen-signaling pathway [29]. Although we previously 
demonstrated an effect of estrogen-signaling on chondrosarcoma cell proliferation, as compared to estrogendependent breast cancer cell line ZR-75-1 the effects in chondrosarcoma, if present, were very subtle. As a positive control, ZR-75-1 showed a $179 \%$ increase of proliferation upon addition of $1 \mathrm{nM} 17 \beta$-estradiol, confirming a functional experimental setup, versus a previously demonstrated 55\% increase in chondrosarcoma proliferation [11] and no significant increase in the current study. Both studies clearly indicate that, in contrast to estrogen-dependent breast cancer, chondrosarcoma proliferation is not fully dependent on estrogens.

Besides investigating estrogen dependence, we tested aromatase inhibitors which block estrogen production, and the effects of tamoxifen and fulvestrant which abrogate estrogen receptor function [30,31]. In the estrogendependent ZR-75-1 breast-cancer cell line proliferation was completely inhibited upon addition of tamoxifen and fulvestrant (Figure 2B). However, in the chondrosarcoma cell cultures, estrogen-signaling inhibition caused no effects on cell proliferation, suggesting that the mechanism driving proliferation in chondrosarcoma is different from the mechanism active in estrogen-dependent breast cancer. In chondrosarcoma, effects of estrogen are much more subtle and likely depend on the tissue culture conditions used, resulting in either marginal effects (in our previous study) or no effects at all.

In addition, the median time to progression in the clinical series was five months both before and after treatment. Therefore, we can conclude that aromatase inhibition was not effective in five conventional chondrosarcoma patients, nor in a patient with dedifferentiated chondrosarcoma. Although a formal prospective phase II trial would have been more suitable to prove (in)efficacy of this concept, we were not able to gain industry support without stronger preclinical data.

Since our study is limited to the effects of estrogen signaling on conventional central chondrosarcoma only, no conclusions can be drawn about the effects of estrogen signaling in the other chondrosarcoma subtypes. However, although we demonstrated the presence of aromatase and ESR1 in a majority of various chondrosarcoma subtypes, our in vitro data on conventional chondrosarcoma and our patient trial including one dedifferentiated chondrosarcoma patient suggest that effects of estrogen-signaling inhibition in other chondrosarcoma subtypes, if present at all, will be very small and that estrogen-signaling inhibition is unlikely to play a major role in chondrosarcoma management.

\section{Conclusions}

In summary, we demonstrated the presence of the components involved in estrogen signaling in a large majority of chondrosarcomas. However, we could not demonstrate a significant effect of estrogen or inhibitors of estrogen signaling on cell proliferation and viability in vitro using central chondrosarcoma cell lines and primary cultures. Despite the previously presented and currently confirmed biological rationale, our in vitro and pilot clinical data suggest that an active estrogen-signaling pathway might just not play a pivotal role in the development and progression of conventional chondrosarcoma and do not support the further development of therapeutic strategies including inhibition of estrogen signaling in chondrosarcoma.

\section{Acknowledgements and funding}

We thank B.E. van den Akker, J.J. Baelde, R. Vossen, M.A.J.H. van Ruler, S. Romeo, I.H. Briaire-de Bruin, and K.G. van der Ham for excellent technical assistance, J. Oosting for help with data analysis, and T. Krenács for expert assistance in TMA construction. N. Athanasou, A.M. Flanagan, P. Picci, S. Daugaard, B. Liegl-Atzwanger, A. Leithner, and the Institute of Orthopaedics, UCL at the Royal National Orthopaedic Hospital are acknowledged for providing tumor tissues and clinical data. M. Namba, T. Kalinski, J.A. Block, J. Trapman, and L.C.J. Dorssers are thanked for providing cell lines OUMS27, C3842, JJ012, LNCaP, and ZR-75-1, respectively. DM and all experiments were funded by the Dutch Cancer Society, project no UL 2007-3815 and the EuroBoNeT consortium [018814], a European Commission granted Network of Excellence for studying the pathology and genetics of bone tumors. $\mathrm{HG}$, AMCJ and PCWH were funded by the LUMC, and JVMGB was funded by the Netherlands Organization for Scientific Research (917-76-315).

\section{Author details}

'Department of Pathology, Leiden University Medical Center, Leiden, The Netherlands. ${ }^{2}$ Department of Clinical Oncology, Leiden University Medica Center, Leiden, The Netherlands. ${ }^{3}$ Department of Tissue Regeneration, MIRA Institute for Biomedical Technology and Technical Medicine, University of Twente, Enschede, The Netherlands.

\section{Authors' contributions}

DM carried out the experiments and drafted the manuscript. HG carried out the pilot patient study. MK, AMCJ and PCWH participated in the design of the study, the interpretation of data, and revision of the manuscript. JVMGB conceived of the study, and participated in its design and coordination, and helped to draft the manuscript. All authors read and approved the final manuscript.

\section{Competing interests}

The authors declare that they have no competing interests.

Received: 8 February 2011 Accepted: 25 July 2011

Published: 25 July 2011

\section{References}

1. Gelderblom H, Hogendoorn PCW, Dijkstra SD, van Rijswijk CS, Krol AD, Taminiau AH, et al: The clinical approach towards chondrosarcoma. Oncologist 2008, 13:320-329.

2. Bovee JV, Hogendoorn PC, Wunder JS, Alman BA: Cartilage tumours and bone development: molecular pathology and possible therapeutic targets. Nat Rev Cancer 2010, 10:481-488.

3. Schrage YM, Machado I, Meijer D, Briaire-de B, van den Akker BE, Taminiau AH, et al: COX-2 expression in chondrosarcoma: a role for celecoxib treatment? Eur J Cancer 2010, 46:616-624.

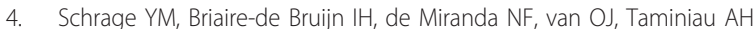
van WT, et al: Kinome profiling of chondrosarcoma reveals SRC-pathway activity and dasatinib as option for treatment. Cancer Res 2009, 69:6216-6222.

5. Evans $\mathrm{HL}$, Ayala $\mathrm{AG}$, Romsdahl MM: Prognostic factors in chondrosarcoma of bone. A clinicopathologic analysis with emphasis on histologic grading. Cancer 1977, 40:818-831. 
6. Milchgrub S, Hogendoorn PCW: Dedifferentiated chondrosarcoma. In World health organization classification of tumours. Pathology and genetics. Tumours of soft tissue and bone Edited by: Fletcher C.D.M., Unni KK, Mertens F 2002, 252-254.

7. Nakashima Y, Park YK, Sugano O: Mesenchymal chondrosarcoma. In World health organization classification of tumours. In Pathology and genetics. Tumours of soft tissue and bone Edited by: Fletcher C.D.M., Unni KK, Mertens F 2002, 255-256.

8. McCarthy EF, Freemont A, Hogendoorn PCW: Clear cell chondrosarcoma. In World health organization classification of tumours. Pathology and genetics. Tumours of soft tissue and bone Edited by: Fletcher C.D.M., Unni KK, Mertens F 2002, 257-258

9. Grumbach MM: Estrogen, bone, growth and sex: a sea change in conventional wisdom. J Pediatr Endocrinol Metab 2000, 13(Suppl 6):1439-1455.

10. Van der Eerden BCJ, Karperien M, Wit JM: The estrogen receptor in the growth plate: implications for pubertal growth. J Pediatr Endocrinol Metab 2001, 14(Suppl 6):1527-1533.

11. Cleton-Jansen AM, van Beerendonk HM, Baelde HJ, Bovée JVMG, Karperien $M$, Hogendoorn PCW: Estrogen signaling is active in cartilaginous tumors: implications for antiestrogen therapy as treatment option of metastasized or irresectable chondrosarcoma. Clin Cancer Res 2005, 11:8028-8035.

12. Grifone TJ, Haupt HM, Podolski V, Brooks JJ: Immunohistochemical expression of estrogen receptors in chondrosarcomas and enchondromas. Int J Surg Pathol 2008, 16:31-37.

13. Odink $A E$, van Asperen CJ, Vandenbroucke JP, Cleton-Jansen AM, Hogendoorn PCW: An association between cartilaginous tumours and breast cancer in the national pathology registration in The Netherlands points towards a possible genetic trait. J Pathol 2001, 193:190-192.

14. Cleton-Jansen AM, Timmerman MC, Van de Vijver MJ, van Asperen CJ, Kroon HM, Eilers $\mathrm{PH}$, et al: A distinct phenotype characterizes tumors from a putative genetic trait involving chondrosarcoma and breast cancer occurring in the same patient. Lab Invest 2004, 84:191-202.

15. Bertoni F, Bacchini P, Hogendoorn PCW: Chondrosarcoma. In World Health Organisation classification of tumours. Pathology and genetics of tumours of soft tissue and bone. Edited by: Fletcher CDM, Unni KK, Mertens F. Lyon: IARC Press; 2002:247-251.

16. Allred DC, Harvey JM, Berardo M, Clark GM: Prognostic and predictive factors in breast cancer by immunohistochemical analysis. Mod Pathol 1998, 11:155-168.

17. Harvey JM, Clark GM, Osborne CK, Allred DC: Estrogen receptor status by immunohistochemistry is superior to the ligand-binding assay for predicting response to adjuvant endocrine therapy in breast cancer. $J$ Clin Oncol 1999, 17:1474-1481.

18. Kunisada T, Miyazaki M, Mihara K, Gao C, Kawai A, Inoue H, et al: A new human chondrosarcoma cell line (OUMS-27) that maintains chondrocytic differentiation. Int J Cancer 1998, 77:854-859.

19. Gil-Benso R, Lopez-Gines C, Lopez-Guerrero JA, Carda C, Callaghan RC, Navarro S, et al: Establishment and characterization of a continuous human chondrosarcoma cell line, ch-2879: comparative histologic and genetic studies with its tumor of origin. Lab Invest 2003, 83:877-887.

20. Jagasia AA, Block JA, Qureshi A, Diaz MO, Nobori T, Gitelis S, et al: Chromosome 9 related aberrations and deletions of the CDKN2 and MTS2 putative tumor suppressor genes in human chondrosarcomas. Cancer Lett 1996, 105:91-103.

21. Dorssers LC, van AT, Dekker A, van Agthoven TL, Kok EM: Induction of antiestrogen resistance in human breast cancer cells by random insertional mutagenesis using defective retroviruses: identification of bcar-1, a common integration site. Mol Endocrinol 1993, 7:870-878.

22. Therasse P, Arbuck SG, Eisenhauer EA, Wanders J, Kaplan RS, Rubinstein L, et al: New guidelines to evaluate the response to treatment in solid tumors. In J Natl Cancer Inst. Volume 92. European Organization for Research and Treatment of Cancer, National Cancer Institute of the United States, National Cancer Institute of Canada; 2000:205-216.

23. Bruns J, Fiedler W, Werner M, Delling G: Dedifferentiated chondrosarcoma-a fatal disease. J Cancer Res Clin Oncol 2005, 131:333-339.

24. Kulyk WM, Franklin JL, Hoffman LM: Sox9 expression during chondrogenesis in micromass cultures of embryonic limb mesenchyme. Exp Cell Res 2000, 255:327-332.
25. Fanburg-Smith JC, Auerbach A, Marwaha JS, Wang Z, Santi M, Judkins AR, et al: Immunoprofile of mesenchymal chondrosarcoma: aberrant desmin and EMA expression, retention of INI1, and negative estrogen receptor in 22 female-predominant central nervous system and musculoskeletal cases. Ann Diagn Pathol 2010, 14:8-14.

26. Fasco MJ, Amin A, Pentecost BT, Yang Y, Gierthy JF: Phenotypic changes in MCF-7 cells during prolonged exposure to tamoxifen. Mol Cell Endocrinol 2003, 206:33-47.

27. Katzenellenbogen BS, Kendra KL, Norman MJ, Berthois Y: Proliferation, hormonal responsiveness, and estrogen receptor content of MCF-7 human breast cancer cells grown in the short-term and long-term absence of estrogens. Cancer Res 1987, 47:4355-4360.

28. Reddel RR, Alexander IE, Koga M, Shine J, Sutherland RL: Genetic instability and the development of steroid hormone insensitivity in cultured T 47D human breast cancer cells. Cancer Res 1988, 48:4340-4347.

29. van Agthoven $T$, van Agthoven $T L$, Portengen $H$, Foekens JA, Dorssers LC: Ectopic expression of epidermal growth factor receptors induces hormone independence in ZR-75-1 human breast cancer cells. Cancer Res 1992, 52:5082-5088.

30. Howell A: Pure oestrogen antagonists for the treatment of advanced breast cancer. Endocr Relat Cancer 2006, 13:689-706.

31. Lewis JS, Jordan VC: Selective estrogen receptor modulators (SERMs): mechanisms of anticarcinogenesis and drug resistance. Mutat Res 2005, 591:247-263.

doi:10.1186/2045-3329-1-5

Cite this article as: Meijer et al.: Expression of aromatase and estrogen receptor alpha in chondrosarcoma, but no beneficial effect of inhibiting estrogen signaling both in vitro and in vivo. Clinical Sarcoma Research 2011 1:5.

\section{Submit your next manuscript to BioMed Central and take full advantage of:}

- Convenient online submission

- Thorough peer review

- No space constraints or color figure charges

- Immediate publication on acceptance

- Inclusion in PubMed, CAS, Scopus and Google Scholar

- Research which is freely available for redistribution

Submit your manuscript at www.biomedcentral.com/submit
Biomed Central 